\title{
Implication of Dark Matter Content in Dwarf Spheroidal Galaxies
}

\author{
H. Hirashita ${ }^{1}$ and T.T. Takeuchi ${ }^{1}$ \\ Department of Astronomy, Faculty of Science, Kyoto University, \\ Sakyo-ku, Kyoto 606-8502, Japan \\ H. Kamaya ${ }^{1}$ \\ Department of Physics, Faculty of Science, Kyoto University, Sakyo-ku, \\ Kyoto 606-8502, Japan
}

\begin{abstract}
We investigate the dark matter (DM) content in the Local Group dwarf spheroidal galaxies (dSphs) by examining the correlations among their physical quantities. Two origins of the large velocity dispersions of the dSphs are possible: [1] the existence of DM and [2] tidal heating by the Galaxy. The correlation tests support both [1] and [2]. We finally mention circumstantial evidence for the existence of DM in the dSphs.
\end{abstract}

\section{Introduction}

Recent observations have been revealing the properties of the Local Group dwarf spheroidal galaxies (dSphs). The dSphs have luminosities of $10^{5}-10^{7} L_{\odot}$, and are characterized by their low surface brightnesses (Gallagher \& Wyse 1994 for a review). In the context of the cold dark matter (CDM) Universe, low mass galaxies are considered to be the first bound luminous objects. Thus, the dSphs are expected to contain the CDM (see also Flin 1998 for an observational approach).

To date, stellar velocity dispersions of $\mathrm{dSphs}$ have been extensively measured (e.g., Mateo et al. 1993). In general, the velocity dispersions indicate too large a mass to be accounted for by the luminous mass in these systems. In other words, dSphs have generally high mass-to-light ratios $(M / L s)$. However, this argument may be challenged if we take into account the tidal force by the Galaxy. If a dwarf galaxy orbiting the Galaxy is significantly perturbed by Galactic tides, the observed velocity dispersion of the dwarf galaxy can be larger than the gravitational equilibrium dispersion (e.g., Kuhn \& Miller 1989).

We will consider whether the observed large $M / L \mathrm{~s}$ of $\mathrm{dSphs}$ are due to the tidal effect or the existence of the DM potentials.

\footnotetext{
${ }^{1}$ Research Fellow of Japan Society for the Promotion of Science
} 


\section{Correlation Among Observed Quantities}

\subsection{Surface Brightness and Galactocentric Distance}

In this section, we consider what determines physical condition of the dSphs. We re-examine the correlation which Bellazzini et al. (1996; hereafter B96) presented.

First, a dimensionless form of the Galactic tidal force is defined. The Galactic potential is modeled by a spherical dark halo with a flat rotation curve of amplitude $V_{\text {rot }}=220 \mathrm{~km} \mathrm{~s}^{-1}$. We assume that the dark halo extends up to $R_{\mathrm{GC}}=100 \mathrm{kpc}$. With this flat rotation model of the Galactic potential, the mass of the Galaxy within the galactocentric distance $R_{\mathrm{GC}}$ is expressed as

$$
\begin{aligned}
& M_{\mathrm{G}}\left(R_{\mathrm{GC}}\right) \\
& = \begin{cases}\frac{V_{\mathrm{rot}}^{2} R_{\mathrm{GC}}}{G}=1.1 \times 10^{12} M_{\odot}\left(\frac{R_{\mathrm{GC}}}{100 \mathrm{kpc}}\right) & \text { for } R_{\mathrm{GC}} \leq 100 \mathrm{kpc}, \\
1.1 \times 10^{12} M_{\odot} & \text { for } R_{\mathrm{GC}} \geq 100 \mathrm{kpc} .\end{cases}
\end{aligned}
$$

The tidal force which a spherical satellite galaxy orbiting the Galaxy experiences is approximately $F_{\mathrm{T}} \equiv G M_{\mathrm{s}} M_{\mathrm{G}} r_{\mathrm{c}} / R_{\mathrm{GC}}^{3}$, while the gravitational binding force of the satellite galaxy is approximately described as $F_{\mathrm{B}} \equiv G M_{\mathrm{s}}^{2} / r_{\mathrm{c}}^{2}$, where $M_{\mathrm{s}}$ and $r_{\mathrm{c}}$ are the total mass and the core radius of the satellite, respectively. We, then, define the dimensionless tidal force identical to the definition by B96:

$$
F_{\mathrm{T}, \mathrm{B}} \equiv \frac{F_{\mathrm{T}}}{F_{\mathrm{B}}}=\left(\frac{M_{\mathrm{G}}}{M_{\mathrm{s}}}\right)\left(\frac{r_{\mathrm{c}}}{R_{\mathrm{GC}}}\right)^{3} .
$$

We estimate $M_{\mathrm{S}}$ in the following two different ways:

[1] Tidal model: The large velocity dispersion of dSphs is attributed not to deep DM potentials but to significant perturbation by Galactic tides.

[2] DM model: dSphs contain significant amount of DM and are virialized in the DM potentials.

For the tidal model, the mass of every $\mathrm{dSph}$ is estimated in the following way:

$$
M_{\mathrm{s}}=M_{\mathrm{L}} \quad(\text { for the tidal model), }
$$

where $M_{\mathrm{L}}$ is the luminous mass calculated directly from the luminosity by assuming $M_{\mathrm{L}} / L=1$ (in solar unit). The difference in the value of $M_{\mathrm{L}} / L=1$ does not affect the analyses hereafter. For the DM model,

$$
M_{\mathrm{s}}=M_{\mathrm{vir}} \quad(\text { for the DM model), }
$$

where $M_{\text {vir }}$ is the virial mass derived from the observed central velocity dispersion by assuming virial equilibrium (Pryor \& Kormendy 1990).

Figure 1 shows the correlation between $\mu_{V}(0)$ (central surface brightness in $V$ band) and $\log F_{\mathrm{T}, \mathrm{B}}$ for the tidal model. The correlation coefficient is $r=0.91$ for the data points, which indicates strong correlation. Thus, B96 insisted that the Galactic tidal effect had settled the present ranking in the dSph central brightness. We have confirmed this result.

Here, the DM model is tested by the same method as B96 except the mass estimations. We derive the mass by using the virial theorem; $M_{\mathrm{s}}=M_{\mathrm{vir}}$. Figure 


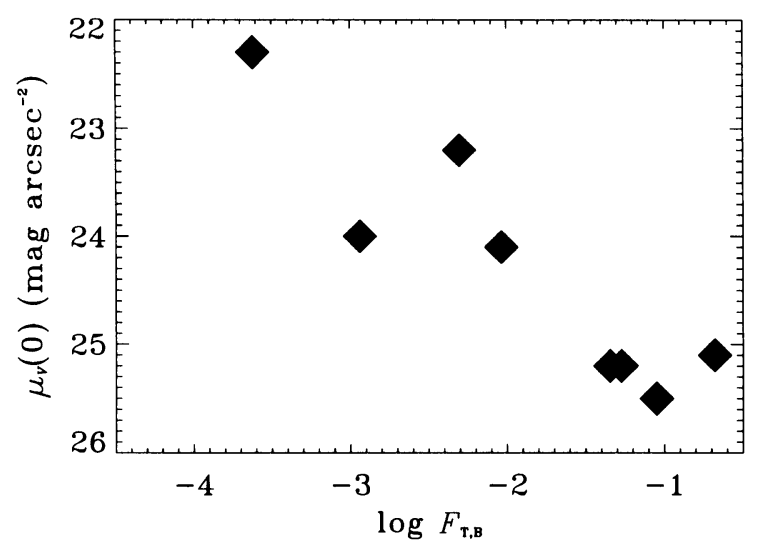

Figure 1. Correlation between $\mu_{V}(0)$ (central surface brightness in the $V$ band) and $F_{\mathrm{T}, \mathrm{B}}$ (dimensionless tidal force) for the tidal model. In this model, the mass of each $\mathrm{dSph}$ is estimated by luminous mass. The correlation coefficient is 0.91 .

2 shows the relation between $\mu_{V}(0)$ and $F_{\mathrm{T}, \mathrm{B}}$ for the DM model. In this model, we find that the two quantities are less correlated $(r=0.48)$ than in the tidal model. The lower correlation is naturally expected for the DM model, since it is $\mathrm{DM}$ in a dSph, not tidal force by the Galaxy, that determines the dynamical condition of the dSphs.

Thus, there is nothing to choose between the two models in the $\mu_{V}(0)-F_{\mathrm{T}, \mathrm{B}}$ relations. We, therefore, need further considerations to determine which of the two models is applicable for dSphs.

\subsection{Tidal Force and Galactocentric Distance}

In this subsection, we present correlation between the dimensionless tidal force and the Galactocentric distance for each model. First, the $\log F_{\mathrm{T}, \mathrm{B}}-\log R_{\mathrm{GC}}$ relation is plotted in Fig. 3 by using the tidal model $\left(M_{\mathrm{S}}=M_{\mathrm{L}}\right)$. In this model, the correlation coefficient is $r=-0.94$. We obtain a strong correlation between the two quantities. Next, the same relation is presented in Fig. 4 by using the DM model $\left(M_{\mathrm{s}}=M_{\mathrm{vir}}\right)$. The two quantities are marginally correlated $(r=-0.68)$.

In the tidal model, the correlation is stronger than in the DM model. Since we determine the mass of a dSph by luminosity in the tidal model, this good correlation can reflect the correlation between luminosity and the Galactocentric distance. Indeed, B96 (their Fig. 1) showed that $\mu_{V}(0)$ and $R_{\mathrm{GC}}$ are significantly correlated. This shows that the good correlation between $F_{\mathrm{T}, \mathrm{B}}$ and $R_{\mathrm{GC}}$ has only a secondary meaning, since the dependence of the luminosity on the Galactocentric distance produces this correlation.

In the DM model, the regression means that

$$
F_{\mathrm{T}, \mathrm{B}} \propto R_{\mathrm{GC}}^{-1.50 \pm 0.40} .
$$




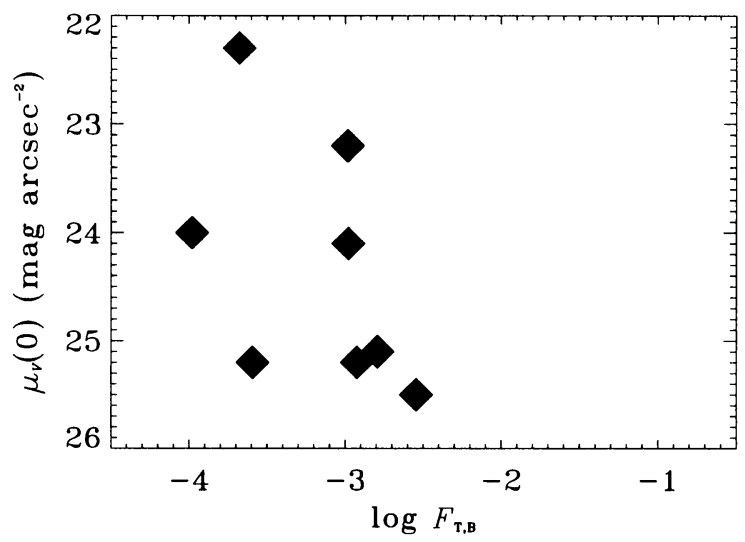

Figure 2. The same as Fig. 1, but for the DM model. The mass of each $\mathrm{dSph}$ is estimated by virial mass. The correlation coefficient is 0.48 .

If the flat rotation curve of the Galaxy is taken into account, $M_{\mathrm{G}} \propto R_{\mathrm{GC}}$ (Eq. 1). Using equation (2), we obtain

$$
F_{\mathrm{T}, \mathrm{B}} \propto \rho_{\mathrm{s}}^{-1} R_{\mathrm{GC}}^{-2}
$$

where we define the mass density of the satellite dwarf galaxies as $\rho_{\mathrm{s}}=M_{\mathrm{s}} / r_{\mathrm{c}}^{3}$. If the density of a dSph is determined by the DM contained in itself, $\rho_{\mathrm{s}}$ is independent of $R_{\mathrm{GC}}$. Using (5) and (6), we obtain

$$
\rho_{\mathrm{s}} \propto R_{\mathrm{GC}}^{-0.50 \pm 0.40} .
$$

This shows that it cannot be determined whether $\rho_{\mathrm{s}}$ depends on $R_{\mathrm{GC}}$ strongly or weakly. We need more sample galaxies to judge whether the density of the $\mathrm{dSphs}$ is internally or environmentally determined.

Thus, as shown in this section, we have no evidence which model is more suitable for a kinematical picture of the dSphs. This implies that the correlation study of the physical quantities of the dSphs is unable to prove the existence of DM in the dSphs.

\section{Discussions}

\subsection{Tidal origin of the large velocity dispersions?}

Piatek \& Prior (1995) showed that the velocity dispersions of dSphs are little affected by tidal force, though tidal force effectively disrupts the outer regions of dSphs. Moreover, the escape timescale, $t_{\mathrm{esc}}$, defined by the time needed for the tidally heated star to escape to $l=1 \mathrm{kpc}$ from a dSph, is estimated as

$$
t_{\mathrm{esc}}=10^{8}\left(\frac{l}{1 \mathrm{kpc}}\right)\left(\frac{v_{\mathrm{esc}}}{10 \mathrm{~km} \mathrm{~s}^{-1}}\right)^{-1} \mathrm{yr},
$$




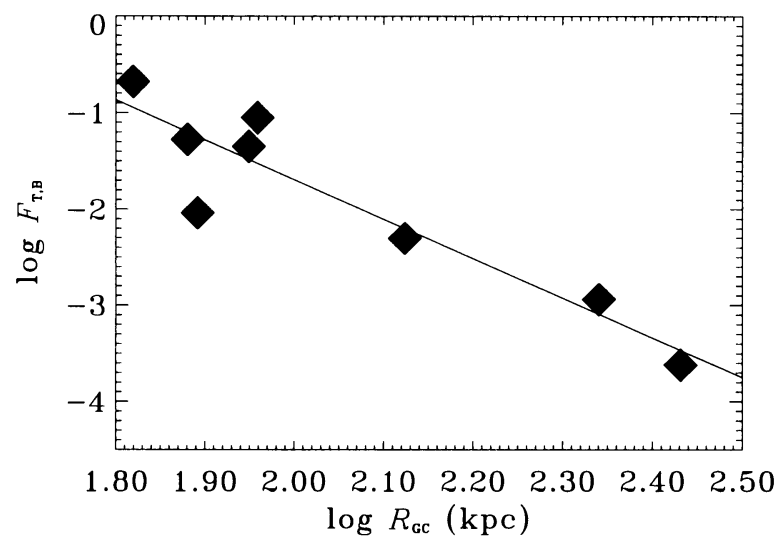

Figure 3. Correlation between $F_{\mathrm{T}, \mathrm{B}}$ (dimensionless tidal force) and $R_{\mathrm{GC}}$ (Galactocentric distance) for the tidal model. The correlation coefficient is -0.94 . The line represents the linear regression.

where $v_{\text {esc }}$ is the escape velocity of the dSph. This estimate is consistent with Johnston (1998). This is an order of magnitude smaller than the orbital timescale of dSphs. Thus, the significantly tidally-perturbed stars in dSphs go their separate ways and cannot be observed as an assembly. Thus, we conclude that tidal disruption seems to be unlikely to explain the high velocity dispersions of the dSphs.

\section{2. $\quad D M$ in dSphs}

[1] Considering that tidally disrupted dSphs survive only $\sim 10^{8} \mathrm{yr}(\S 3.1)$, the DM content in dSphs is needed to bind a dSph as a system. As implied in Hirashita et al. (1998; see also Einasto et al. 1974, van den Bergh 1994 and Hirashita et al. 1997), ram-pressure stripping by the Galactic gaseous halo may be effective for the determination of the physical parameters of the dSphs. Even in this context, the DM content is necessary to maintain the dSphs as a bound system.

[2] In $\S 2$, relation (7) indicates that $\rho_{\mathrm{s}}$ may correlate little with the Galactocentric distance. Since this implies that the density is determined by the internal structure of the dSphs, this is consistent with the DM-dominant picture of dSphs.

Acknowledgements. We thank S. Mineshige for continuous encouragement. We also thank S. van den Bergh and T.E. Armandroff for useful comments on the dwarf spheroidal galaxies. We are grateful to T.T. Ishii for kind help. All the authors acknowledge the Research Fellowship of the Japan Society for the Promotion of Science for Young Scientists. 


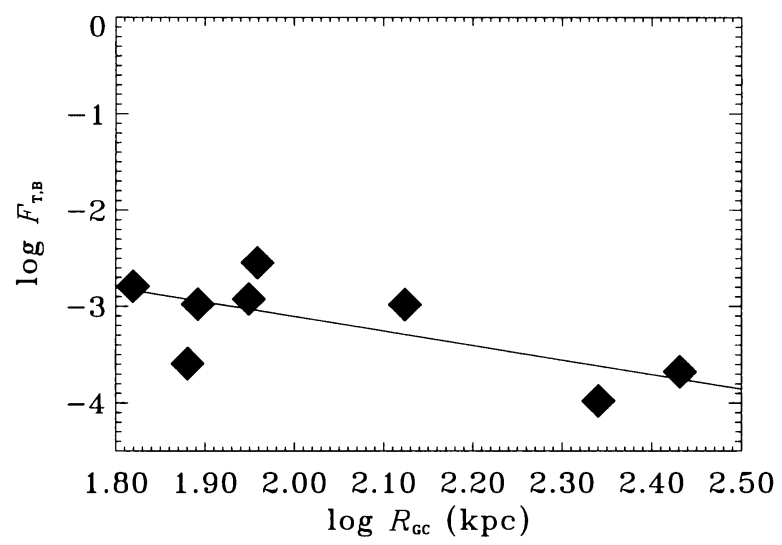

Figure 4. The same as Fig. 3, but for the DM model. The correlation coefficient is -0.68 .

\section{References}

Bellazzini, M., Fusi Pecci, F., Ferraro, F.R. 1996, MNRAS, 278, 947 (B96)

Einasto, J., Saar, E., Kaasik, A. 1974, Nature, 252, 111

Flin, P. 1998, these proceedings

Gallagher, J.S., Wyse, R.F.G. 1994, PASP, 106, 1225

Hirashita, H., Kamaya, H., Mineshige, S. 1997, MNRAS, 290, L33

Hirashita, H., Kamaya, H., Takeuchi, T.T. 1998, ApJ, submitted

Johnston, K.V. 1998, ApJ, 495, 297

Kuhn J.R., Miller R.H. 1989, ApJ, 341, L41

Mateo, M., Olzewski, E.W., Pryor, C., Welch, D.L., Fischer, P. 1993, AJ, 105, 510

Piatek, S., Pryor, C. 1995, AJ, 109, 1071

Pryor, C., Kormendy, J. 1990, AJ, 100, 127

van den Bergh, S. 1994, ApJ, 428, 617

\section{Discussion}

Armandroff: In order to try to resolve the question of whether the dark matter model or the tidal model is correct, one can compare the predictions of the dynamical modelling with observations. For example, the tidal disruption simulations of Piatek \& Pryor and also Oh, Lin \& Aarseth (ApJ 442, 1420, 1995) find that the velocity dispersion remains small, but relatively large streaming motions dominate the kinematics. These streaming motions have been searched for in Galactic dSphs, but have not been found (see the study of Draco \& Ursa Minor by Pryor, Olszewski \& Armandroff; AJ 111, 750, 1996 and refs. therein). Also, the Kroupa modelling (ApJ 498, 143, 1998 and refs therein) predicts that 
tidal dwarfs will be quite elongated along the line of sight. Such line-of-sight elongation should be observable via substantial scatter in the magnitudes of the horizontal branch stars. As far as I have been able to investigate, the small scatter in the horizontal branch magnitudes of Galactic dSphs with the best data rules out substantial elongation along the line of sight. Thus, the tidal model faces two challenges. 